\section{Association of Parvovirus B19 Infection with Systemic Lupus Erythematosus: Role of Th1 Predominance}

To the Editor:

We read with interest the article by Hession, et $a l^{1}$ that described a 59-year-old woman who developed systemic lupus erythematosus (SLE) after parvovirus B19 infection; they wondered whether this phenomenon might be due to clinical mimicry or autoimmune induction ${ }^{1}$.

Although not extensively studied to date, some reports show Th1-type cytokines might be increased during parvovirus B19 infection ${ }^{2}$. Isa, et al reported that elevated levels of the Th1 cytokines such as interleukin 12 (IL-12) and IL-15 were evident at the time of the initial peak of parvovirus B19 viral load during acute infection, and some of these patients had a sustained Th1 cytokine response during followup (20 to 130 weeks after acute infection) $)^{2}$. They also showed that the Th1 cytokine response correlated with the previously identified sustained CD8+ $\mathrm{T}$ cell response and viremia ${ }^{2}$.

Also, there have been reports showing Th1-type cytokines might be involved in the development of systemic lupus erythematosus (SLE) ${ }^{3,4}$. Tokano, $e t a l^{3}$ reported that the levels of IL-12 in SLE patients were significantly higher than those in normal subjects; Aringer, et $a l^{4}$ showed that IL-15 was elevated in SLE sera and was correlated significantly with Bcl-2 expression. Segal, et $\mathrm{l}^{5}$ reported that administration of IL-12 to aging mice reversed their Th1/Th2 cytokine profile and thus rendered them vulnerable to induction of experimental SLE; and Bo, et $a l^{6}$ demonstrated that elevated expression of transmembrane IL-15 in immune cells correlated with the development of murine lupus, suggesting that Th1-associated cytokines might play a crucial role in the pathogenesis and development of SLE.

Thus there is a possibility that persistently increased Th1-associated cytokines, especially IL-12 and IL-15, after parvovirus B19 infection might be involved not only in transient symptoms of SLE but also in chronic SLE autoimmunity. Further studies should be performed to evaluate whether Th1-associated cytokine levels might correlate with the $\operatorname{IgM} / \operatorname{IgG}$ antibody titers or viremia in parvovirus B19 infection-associated SLE.
SE JIN PARK, MD; JI HONG KIM, MD, The Institute of Kidney Disease, Department of Pediatrics, Yonsei University College of Medicine, Severance Children's Hospital, Seoul; TAE SUN HA, MD, Department of Pediatrics, Chungbuk National University College of Medicine, Cheongju; JAE IL SHIN, MD, The Institute of Kidney Disease,

Department of Pediatrics, Yonsei University College of Medicine, Severance Children's Hospital, Seoul, Korea.

Address correspondence to Dr. J.I. Shin, Department of Pediatrics, Yonsei University College of Medicine, Sungsan-Ro 250, Seodaemun-Ku, 120-752, CPO Box 8044, Seoul, Korea. E-mail: shinji@yuhs.ac

\section{REFERENCES}

1. Hession MT, Au SC, Gottlieb AB. Parvovirus B19-associated systemic lupus erythematosus: clinical mimicry or autoimmune induction? J Rheumatol 2010;37:2430-2.

2. Isa A, Lundqvist A, Lindblom A, Tolfvenstam T, Broliden $\mathrm{K}$. Cytokine responses in acute and persistent human parvovirus B19 infection. Clin Exp Immunol 2007;147:419-25.

3. Tokano Y, Morimoto S, Kaneko H, Amano H, Nozawa K, Takasaki Y, et al. Levels of IL-12 in the sera of patients with systemic lupus erythematosus (SLE) - relation to Th1- and Th2-derived cytokines. Clin Exp Immunol 1999;116:169-73.

4. Aringer M, Stummvoll GH, Steiner G, Köller M, Steiner CW, Höfler E, et al. Serum interleukin-15 is elevated in systemic lupus erythematosus. Rheumatology 2001;40:876-81.

5. Segal R, Dayan M, Zinger H, Habut B, Shearer GM, Mozes E. The effect of IL-12 on clinical and laboratory aspects of experimental SLE in young and aging mice. Exp Gerontol 2003;38:661-8.

6. Bo H, Wei XQ, Dong H, Zhang Y, Lv P, Liu W, et al. Elevated expression of transmembrane IL-15 in immune cells correlates with the development of murine lupus: a potential target for immunotherapy against SLE. Scand J Immunol 2009;69:119-29.

J Rheumatol 2011;38:6; doi:10.3899/jrheum.101332 